\title{
EXTREME EIGENVALUES OF TRANSLATION KERNELS
}

\author{
BY \\ HAROLD WIDOM
}

1. Let $\rho$ be an even real-valued function belonging to $L_{1}(-\infty, \infty)$ and consider the integral equation

$$
A \int_{-1}^{1} \rho(A(x-y)) \psi(y) d y=\mu \psi(x), \quad|x|<1 .
$$

Denote the positive eigenvalues of (1) by

$$
\mu_{1, A} \geqq u_{2, A} \geqq \cdots
$$

and let $\psi_{j, A}$ be a normalized eigenfunction corresponding to the eigenvalue $\mu_{j, A}$. It is the purpose of this paper to determine the behavior of $\mu_{j, A}$ and $\psi_{j, A}$ as $A \rightarrow \infty$ (with fixed $j=1,2, \cdots$ ), of course with appropriate conditions imposed on $\rho$.

Set

$$
R(\xi)=\int_{-\infty}^{\infty} e^{i \xi x} \rho(x) d x .
$$

It was proved in [4] that if $R(\xi)$ is sufficiently smooth, assumes its maximum $M$ at $\xi=0$ and only at $\xi=0$, and

$$
\lim _{\xi \rightarrow 0} \xi^{-2}(M-R(\xi))=c
$$$$
(0<c<\infty)
$$

then

$$
\lim _{A \rightarrow \infty} A^{2}\left(M-\mu_{j, A}\right)=c \pi^{2} j^{2} / 4 .
$$

This result had been conjectured in [1], where the discrete analogue (concerning Toeplitz matrices) was proved. In [5] we considered the case where (3) was replaced by

$$
\lim _{\xi \rightarrow 0}|\xi|^{-\alpha}(1-R(\xi))=c \quad(0<c<\infty),
$$

with $\rho$ a probability density, so that of necessity $0<\alpha \leqq 2$. It was shown that

Received by the editors January 30, 1961. 


$$
\lim _{A \rightarrow \infty} A^{\alpha}\left(1-\mu_{j, A}\right)=c \lambda_{j}^{-1}
$$

where

$$
\lambda_{1} \geqq \lambda_{2} \geqq \cdots
$$

are the eigenvalues of a certain positive definite kernel $K(x, y)$ on $(-1,1)$. (The kernel of course depends on $\alpha$. It is given by (6) below.) Moreover any sequence of $A$ 's tending to infinity has a subsequence for which $\psi_{j, A}$ converges in mean square to an eigenfunction of $K$ corresponding to the eigenvalue $\boldsymbol{\lambda}_{j}$.

When $\alpha=2$

$$
K(x, y)=\frac{1}{2}[1+\min (x, y)][1-\max (x, y)]
$$

which is the Green's function for the operator $u \rightarrow u^{\prime \prime}$ with boundary conditions $u(-1)=u(1)=0$. When $\alpha=1$ the kernel is again elementary. It is

$$
\frac{1}{2 \pi} \log \frac{1-x y+\left[\left(1-x^{2}\right)\left(1-y^{2}\right)\right]^{1 / 2}}{1-x y-\left[\left(1-x^{2}\right)\left(1-y^{2}\right)\right]^{1 / 2}} .
$$

The theorem in this case had also been conjectured in [1].

In this note we remove the restriction $\alpha \leqq 2$. We take as our starting point a bounded real-valued function $R(\xi)$ and consider the operator $T_{A}$ on $L_{2}(-1,1)$ defined by

$$
T_{A} \psi(x)=\frac{1}{(2 \pi)^{1 / 2}} \int_{-\infty}^{\infty} e^{-i \xi x} R\left(\frac{\xi}{A}\right) \hat{\psi}(\xi) d \xi, \quad|x|<1,
$$

where

$$
\hat{\psi}(\xi)=\frac{1}{(2 \pi)^{1 / 2}} \int_{-1}^{1} e^{i \xi x} \psi(x) d x .
$$

We assume that for each $A$ this operator is completely continuous. This will hold if

$$
\lim _{\xi \rightarrow \pm \infty} R(\xi)=0
$$

or if (2) holds for some $\rho \in L_{p}(1 \leqq p \leqq 2)$. In the latter case $T_{A}$ is the integral operator with kernel $A \rho(A(x-y))$ and we are dealing with the equation (1).

The additional assumptions on $R$ are:

$$
\begin{aligned}
\max _{|\xi|>\delta} R(\xi)<M & \text { (for each } \delta>0), \\
\lim _{\xi \rightarrow 0}|\xi|^{-\alpha}(M-R(\xi))=c & (0<c<\infty, 0<\alpha<\infty) .
\end{aligned}
$$


We shall produce a positive definite kernel $K(x, y)$ whose eigenvalues we denote by $\lambda_{1} \geqq \lambda_{2} \geqq \cdots$, and shall prove the following:

THEOREM. Denote the positive eigenvalues of $T_{A}$ by $\mu_{1, A} \geqq \mu_{2, A} \geqq \cdots$ and let $\psi_{j, A}$ be a normalized eigenfunction corresponding to $\mu_{j, A}$. Then for fixed $j$

$$
\lim _{A \rightarrow \infty} A^{\alpha}\left(M-\mu_{j, A}\right)=c \lambda_{j}^{-1} .
$$

Moreover each sequence of $A$ 's tending to infinity has a subsequence for which $\psi_{j, A}$ converges in mean square to an eigenfunction of $K$ belonging to the eigenvalue $\lambda_{j}$.

In case $\alpha$ is an even integer $2 k$ the kernel $K(x, y)$ will be the Green's function for the operator $u \rightarrow(-1)^{k} u^{(2 k)}$ on $(-1,1)$ with boundary conditions $u^{(l)}(-1)=u^{(l)}(1)=0,0 \leqq l \leqq k-1$. (This follows from Lemma 3 below.) In this case the Toeplitz matrix analogue of the theorem was proved by Parter [3]. In his proof Parter observed that it is enough to show that the limit of the eigenfunctions is a weak eigenfunction of the formal limit. We use this idea in our proof.

We shall repeat here a few things already done in [5], notably the derivation of certain identities involving $K$. This permits the present paper to be entirely self-contained.

2. Define, for $x, y \in(-1,1)$ and $0<\Re \alpha<2$,

$$
\begin{aligned}
K(x, y)=\frac{\cos \alpha \pi / 2}{\Gamma(\alpha)} & |x-y|^{\alpha-1} \max (0, x-y) \\
& -\frac{\sin \alpha \pi / 2}{\pi \Gamma(\alpha)}\left(1-y^{2}\right)^{\alpha / 2} \int_{-1}^{x} \frac{(x-t)^{\alpha-1}}{\left(1-t^{2}\right)^{\alpha / 2}(t-y)} d t .
\end{aligned}
$$

The integral is to be interpreted as a principal value if $y<x$. If $R \alpha \leqq 1$, $K(x, y)$ is defined only for $x \neq y$.

Define, for $|y|<1$, all real $x$, and $0<R \alpha<2(\alpha \neq 1)$,

$$
\begin{aligned}
K_{0}(x, y)= & \frac{\sec \alpha \pi / 2}{2 \Gamma(\alpha)}|x-y|^{\alpha-1} \\
& -\frac{\tan \alpha \pi / 2}{2 \pi \Gamma(\alpha)}\left(1-y^{2}\right)^{\alpha / 2} \int_{|t|>1} \frac{|t-x|^{\alpha-1}}{\left(t^{2}-1\right)^{\alpha / 2}|t-y|} d t .
\end{aligned}
$$

LEMma 1. We have

$$
K_{0}(x, y)=\left\{\begin{array}{cl}
K(x, y), & |x| \leqq 1 \\
0, & |x| \geqq 1 .
\end{array}\right.
$$

Proof. Assume first that $\alpha$ is real. The function 


$$
\frac{(t-x)^{\alpha-1}}{\left(t^{2}-1\right)^{\alpha / 2}}
$$

is analytic in $g t>0$, all relevant arguments being taken to lie between 0 and $\pi$. If we extend the function to the real axis by continuity, a simple contour integration shows

$$
\int_{-\infty}^{\infty} \frac{(t-x)^{\alpha-1}}{\left(t^{2}-1\right)^{\alpha / 2}(t-y)} d t=\pi i(y-x)^{\alpha-1}\left(y^{2}-1\right)^{-\alpha / 2} .
$$

Assume $x \geqq 1$. Then (8) may be written

$$
\begin{aligned}
\left\{-\int_{-\infty}^{-1}-e^{i \pi \alpha / 2} \int_{-1}^{1}-e^{i \pi \alpha} \int_{1}^{x}+\int_{x}^{\infty}\right\} & \frac{|t-x|^{\alpha-1}}{\left|t^{2}-1\right|^{\alpha / 2}(t-y)} d t \\
& =-\pi i e^{i \pi \alpha / 2}(x-y)^{\alpha-1}\left(1-y^{2}\right)^{-\alpha / 2}
\end{aligned}
$$

If we multiply both sides by $e^{-i \pi \alpha / 2}$ and take imaginary parts, we obtain

$$
\sin \alpha \pi / 2 \int_{|t|>1} \frac{|t-x|^{\alpha-1}}{\left(t^{2}-1\right)^{\alpha / 2}|t-y|} d t=\pi(x-y)^{\alpha-1}\left(1-y^{2}\right)^{-\alpha / 2} .
$$

This shows $K_{0}(x, y)=0$ for $x \geqq 1$. Since $K_{0}(x, y)=K_{0}(-x,-y)$ we also have $K_{0}(x, y)=0$ for $x \leqq-1$. A similar argument applied to (8) with $|x| \leqq 1$ gives $K_{0}(x, y)=K(x, y)$. Finally the identity (7) for real $\alpha$ implies the identity for complex $\alpha$, since both sides are, for fixed $x$ and $y$, analytic in $\alpha$.

We leave to the reader the proof of the estimates

$$
K(x, y) \leqq \begin{cases}B|x-y|^{R^{\alpha-1}}, & \mathbb{R} \alpha<1, \\ B \log \left(1+|x-y|^{-1}\right), & \mathbb{R} \alpha=1, \\ B, & \Re \alpha>1,\end{cases}
$$

Of course $B$ denotes a constant which will depend on $\alpha$.

Lemma 2. For any $\alpha$ in $0<R \alpha<2$ we have

$$
\begin{aligned}
|\xi|^{\alpha} \int_{-1}^{1} K(x, y) e^{i \xi x} d x & =e^{i \xi y} \\
- & \frac{1}{\pi}(\sin \alpha \pi / 2)\left(1-y^{2}\right)^{\alpha / 2} \int_{\mid\{\mid>1} \frac{e^{i \xi t}}{\left(t^{2}-1\right)^{\alpha / 2}|t-y|} d t .
\end{aligned}
$$

Proof. We may assume $R \alpha<1$. We have

$$
\int_{-\infty}^{\infty} e^{i \xi x}|x-y|^{\alpha-1} d x=2 \Gamma(\alpha)(\cos \alpha \pi / 2)|\xi|^{-\alpha} e^{i \xi \nu} .
$$

Also, 


$$
\begin{gathered}
\int_{-X}^{x} e^{i \xi x} d x \int_{|t|>1} \frac{|t-x|^{\alpha-1}}{\left(t^{2}-1\right)^{\alpha / 2}|t-y|} d t \\
\quad=\int_{|t|>1} \frac{e^{i \xi t}}{\left(t^{2}-1\right)^{\alpha / 2}|t-y|} d t \int_{-X-t}^{X-t} e^{i \xi x}|x|^{\alpha-1} d x .
\end{gathered}
$$

As $X \rightarrow \infty$ the inner integral tends boundedly to $2 \Gamma(\alpha)(\cos \alpha \pi / 2)|\xi|^{-\alpha}$. Consequently

$$
\begin{gathered}
\int_{-\infty}^{\infty} e^{i \xi x} d x \int_{|t|>1} \frac{|t-x|^{\alpha-1}}{\left(t^{2}-1\right)^{\alpha-2}|t-y|} d t \\
\quad=2 \Gamma(\alpha)(\cos \alpha \pi / 2)|\xi|^{-\alpha} \int_{|t|>1} \frac{e^{i \xi t}}{\left(t^{2}-1\right)^{\alpha / 2}|t-y|} d t .
\end{gathered}
$$

The identities (7), (10), and (11) give (9).

The analytic continuation of $K$ to $R \alpha>0$ is obtained, when $x<y$, by successive partial integration of the right side of (6). We obtain, for $\alpha=2 k+\beta(k=0,1, \cdots, 0 \leqq \Re \beta<2)$,

$$
\begin{aligned}
K(x, y)=\frac{2^{k} \sin \alpha \pi / 2}{\pi \Gamma(\alpha)(\alpha-2) \cdots(\alpha-2 k)} & \left(1-y^{2}\right)^{\alpha / 2} \\
& \cdot \int_{-1}^{x}(1+t)^{-\beta / 2} \frac{d^{k}}{d t^{k}} \frac{(x-t)^{\alpha-1}}{(1-t)^{\alpha / 2}(y-t)} d t .
\end{aligned}
$$

As for the case $x>y$, note that Lemma 4 below implies that for real $\alpha<2$ we have $K(x, y)=K(y, x)$ for all $x, y$. Thus we may take as the analytic continuation of $K(x, y)$ for $x>y$ the continuation found for $K(y, x)$. The reader can verify that the kernel $K(x, y)$ is bounded if $R \alpha \geqq 2$.

The extension of (9) is found by successive partial integration. We obtain (with $\alpha, \beta$, and $k$ as before)

$$
\begin{aligned}
& |\xi|^{\alpha} \int_{-1}^{1} K(x, y) e^{i \xi x} d x=e^{i \xi y} \\
& \quad-\frac{\sin \alpha \pi / 2}{\pi(\alpha-2) \cdots(\alpha-2 k)}\left(1-y^{2}\right)^{\alpha / 2} \int_{|t|>1}\left(t^{2}-1\right)^{-\beta / 2} \delta^{k} \frac{e^{i \xi t}}{|t-y|} d t
\end{aligned}
$$

where $\delta$ denotes the operation of multiplication by $t^{-1}$ followed by $d / d t$.

We assume henceforth that $\alpha$ is real and positive, and retain the meaning of $k$ and $\beta: k=[\alpha / 2]$ and $\beta=\alpha-2 k$. We denote by $K$ the integral operator on $L_{2}(-1,1)$ with kernel $K(x, y)$.

Lемма 3. If $f \in L_{2}$ then $|\xi|^{\gamma}(K f)^{\frown}(\xi) \in L_{2}$ for any $\gamma$ satisfying 


$$
\begin{aligned}
& 0 \leqq \gamma \leqq \alpha / 2, \\
& 0 \leqq \gamma<(\alpha+\beta) / 2, \\
& 0 \leqq \gamma<(\alpha+1) / 2,
\end{aligned}
$$

$$
\begin{aligned}
\beta & =0, \\
0<\beta<1, & \\
\beta & \geqq 1 .
\end{aligned}
$$

If $g \in L_{2}$ and $|\xi|^{\alpha / 2} \hat{g}(\xi) \in L_{2}$ then

$$
\left(|\xi|^{\alpha}(K f)^{\curlywedge}, \hat{g}\right)=(f, g) .
$$

Proof. From (12) and the definition of $\delta$ we obtain

$$
\begin{aligned}
& |\xi|^{\alpha}(K f) \frown(\xi)=\hat{f}(\xi)-\sum_{l=0}^{k} \sum_{m=0}^{l} a_{l, m} \xi^{l} \int_{-1}^{1}\left(1-y^{2}\right)^{\alpha / 2} f(y) d y \\
& \cdot \int_{|t|>1} e^{i \xi t\left(t^{2}-1\right)^{-\beta / 2} t^{-k-l}} \frac{\operatorname{sgn} t}{(y-t)^{l-m+1}} d t
\end{aligned}
$$

where the $a_{l, m}$ are constants. From Schwarz's inequality

$$
\int_{-1}^{1}\left(1-y^{2}\right)^{\beta / 2} \frac{|f(y)|}{|y-t|} d y \leqq \begin{cases}B & \beta>1, \\ B\left\{\log \left(t^{2}-1\right)^{-1}\right\}^{1 / 2} & \beta=1, \\ B\left(t^{2}-1\right)^{(\beta-1) / 2} & \beta<1,\end{cases}
$$

for $t$ sufficiently close to \pm 1 . It follows that we may apply Fubini's theorem to all the integrals on the right side of (13);

$$
\begin{aligned}
& |\xi|^{\alpha}(K f) \frown(\xi)=\hat{f}(\xi)-\sum_{l=0}^{k} \sum_{m=0}^{l} a_{l, m} \xi^{l}
\end{aligned}
$$

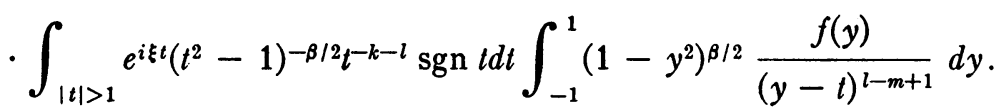

Now it follows from (14) that each of the functions

$$
\left(t^{2}-1\right)^{-\beta / 2} t^{-k-l} \operatorname{sgn} t \int_{-1}^{1}\left(1-y^{2}\right)^{\beta / 2} \frac{f(y)}{(y-t)^{l-m+1}} d y
$$

belongs to $L_{p}$ for any $p$ in

$$
1 \leqq p<\min \left(2, \frac{2}{\beta}\right),
$$

and so [2, Theorem 74] the Fourier transform of each of these functions belong to $L_{q}$ for any $q$ in

$$
\max \left(2, \frac{2}{2-\beta}\right)<q \leqq \infty .
$$

Therefore (15) may be written 


$$
|\xi| \alpha(K f)^{\frown}(\xi)=\hat{f}(\xi)-\left(1+|\xi|^{k}\right) F(\xi)
$$

where $F(\xi) \in L_{q}$. For $\beta>0$ the first part of the lemma follows upon application of Hölder's inequality.

The case $\beta=0$ we treat separately. We obtain from (12) in this case

$$
\begin{aligned}
& |\xi| \alpha \int_{-1}^{1} K(x, y) e^{i \xi x} d x=e^{i \xi y} \\
& \quad+\left.\frac{(-1)^{k}}{2(\alpha-2) \cdots(\alpha-2 k+2)}\left(1-y^{2}\right)^{\alpha / 2} \frac{1}{t} \delta^{k-1} \frac{e^{i \xi t}}{|t-y|}\right|_{t=-1} ^{t=1} .
\end{aligned}
$$

Therefore

$$
|\xi|^{\alpha}(K f) \frown(\xi)=\hat{f}(\xi)+F(\xi)
$$

where $F(\xi) \leqq B|\xi|^{k-1}$, and it is immediate that $|\xi|^{\alpha / 2}(K f)^{-}=|\xi|^{k}(K f)^{-} \in L_{2}$.

As for the second part of the lemma, consider such a function $g$. Then from (15)

$$
\begin{aligned}
(|\xi| \alpha(K f) \uparrow, \hat{g}) & =(\hat{f}, \hat{g})-\sum_{l=0}^{k} \sum_{m=0}^{l} i^{l} a_{l m}\left(g^{(l)} \uparrow(\xi),\right. \\
& \left.\int_{|t|>1} e^{i \xi t}\left(t^{2}-1\right)^{-\beta / 2} t^{-k-l} \operatorname{sgn} t d t \int_{-1}^{1}\left(1-y^{2}\right)^{\beta / 2} \frac{f(y)}{(y-t)^{l-m+1}} d y\right),
\end{aligned}
$$

and it suffices to show that Parseval's identity holds for each term in the summation. As observed above the right side of the inner product is the Fourier transform of an $L_{p}$ function for any $p$ in

$$
1<p<\min \left(2, \frac{2}{\beta}\right) \text {. }
$$

It is sufficient for the validity of Parseval's identity that $g^{(l)}$ - belong to $L_{p}$ for the same $p\left[2\right.$, Theorem 75]. It suffices to show $|\xi|^{k} \hat{g} \in L_{p}$. From the assumption on $g$ and Hölder's inequality it follows that $|\xi|^{k} \hat{g} \in L_{p}$ for any $p$ in

$$
\frac{2}{\beta+1}<p<2
$$

We can always find a $p$ common to the two intervals.

Lemma $4 . K$ is positive definite.

Proof. From Lemma 3 with $g=K f$ we obtain

$$
(f, K f)=\left(|\xi| \alpha\left(K_{J}\right)^{\wedge},(K f)^{\wedge}\right) \geqq 0
$$

so $K$ is positive semi-definite. It remains to prove that $K f=0$ implies $f=0$. 
If $K f=0$ then, again by Lemma 3, we have $(f, g)=0$ for all $g$ 's with $|\xi|^{\alpha / 2} \hat{g}$ $\in L_{2}$. Since the set of such $g$ 's is dense in $L_{2}(-1,1)$ we must have $f=0$.

LEMMA 5. Let $\phi$ be an eigenfunction of $K$ corresponding to the eigenvalue $\lambda$. Then $|\xi|^{\alpha / 2} \hat{\phi}(\xi) \in L_{2} ;$ and if $g \in L_{2}$ is such that $|\xi|^{\alpha / 2} \hat{g} \in L_{2}$ then

$$
(|\xi| \alpha \hat{\phi}, \hat{g})=\lambda^{-1}(\phi, g) \text {. }
$$

Proof. This is an immediate consequence of Lemmas 3 and 4.

3 . In this section we prove the theorem. We assume it true for all $j^{\prime}<j$ and shall deduce it for $j$. For simplicity we take $M=c=1$; the general result follows easily from this special case.

LEMMA 6.

$$
\limsup _{A \rightarrow \infty} A^{\alpha}\left(1-\mu_{j, A}\right) \leqq \lambda_{j}^{-1} .
$$

Proof. It suffices to show that every sequence of $A$ 's tending to infinity has a subsequence for which the inequality holds. For each $A$ of the given sequence let $\psi_{k, A}(k=1, \cdots, j-1)$ be orthonormal eigenfunctions of $T_{A}$ corresponding to the eigenvalues $\mu_{k, A}$. By our induction hypothesis we can find a subsequence of $A$ 's such that each $\psi_{k, A}$ converges in mean square to $\phi_{k}$, an eigenfunction of $K$ corresponding to the eigenvalue $\lambda_{k}$. Now

$$
A^{\alpha}\left(1-\mu_{j, A}\right)=\min \frac{\left(A^{\alpha}\left(I-T_{A}\right) \psi, \psi\right)}{(\psi, \psi)} \quad(I=\text { identity operator })
$$

where the minimum is taken over all $\psi$ orthogonal to each $\psi_{k, A}$. Let $\phi_{j}$ be a normalized eigenfunction of $K$ which corresponds to $\lambda_{j}$ and which is orthogonal to each $\phi_{k}$. (The orthogonality is automatic only if $\lambda_{j}$ has multiplicity one.) Then

$$
\psi(x)=\phi_{j}(x)-\sum_{k=0}^{j-1}\left(\phi_{j}, \psi_{k, A}\right) \psi_{k, A}(x)
$$

is orthogonal to each $\psi_{k, A}$ and we have

$$
\left(A^{\alpha}\left(I-T_{A}\right) \psi, \psi\right)=\left(A^{\alpha}\left(I-T_{A}\right) \phi_{j}, \phi_{j}\right)-\sum A^{\alpha}\left(1-\mu_{k, A}\right)\left(\phi_{j}, \psi_{k, A}\right)^{2} .
$$

Now

$$
\lim _{A \rightarrow \infty} \sum A^{\alpha}\left(1-\mu_{k, A}\right)\left(\phi_{j}, \psi_{k, A}\right)^{2}=\sum \lambda_{k}^{-1}\left(\phi_{j}, \phi_{k}\right)^{2}=0
$$

Next,

$$
\left(A^{\alpha}\left(I-T_{A}\right) \phi_{j}, \phi_{j}\right)=\int_{-\infty}^{\infty} A^{\alpha}\left(1-R\left(\frac{\xi}{A}\right)\right)\left|\hat{\phi}_{j}(\xi)\right|^{2} d \xi .
$$

It follows from (5) that 


$$
A^{\alpha}\left(1-R\left(\frac{\xi}{A}\right)\right) \leqq B|\xi|^{\alpha}
$$

and

$$
\lim _{A \rightarrow \infty} A^{\alpha}\left(1-R\left(\frac{\xi}{A}\right)\right)=|\xi|^{\alpha}
$$

By Lemma 5 we may apply the dominated convergence theorem to the integral in (19) and obtain

$$
\lim _{A \rightarrow \infty}\left(A^{\alpha}\left(I-T_{A}\right) \phi_{j}, \phi_{j}\right)=\int_{-\infty}^{\infty}|\xi|^{\alpha}\left|\hat{\phi}_{j}(\xi)\right|^{2} d \xi=\lambda_{j}^{-1} .
$$

From (17), (18), and (20),

$$
\lim _{A \rightarrow \infty}\left(A^{\alpha}\left(I-T_{A}\right) \psi, \psi\right)=\lambda_{j}^{-1}
$$

Since

$$
\lim _{A \rightarrow \infty}(\psi, \psi)=1-\lim _{A \rightarrow \infty} \sum\left(\phi_{j}, \psi_{k, A}\right)^{2}=1
$$

the lemma is proved.

Lemмa 7. Any sequence of $A$ 's tending to infinity has a subsequence for which

$$
\lim _{A \rightarrow \infty} A^{\alpha}\left(1-\mu_{j, A}\right)=\lambda_{j}^{-1}
$$

and for which $\psi_{j, A}$ converges in mean square to an eigenfunction of $K$ corresponding to $\lambda_{j}$.

Proof. Let $\psi_{k, A}(k=1, \cdots, j-1)$ be normalized eigenfunctions of $T_{A}$ which correspond to $\mu_{k, A}$ and which are orthogonal to $\psi_{j, A}$. Pick a subsequence of $A$ 's such that $\psi_{k, A}$ converge in mean square to $\phi_{k}$ and such that $\psi_{j, A}$ converges weakly to a function $f$. We show that $\psi_{j, A}$ converges in mean square. (This is essentially Lemma 1 of [5]. We repeat the proof here.) It suffices to show $(f, f) \geqq 1$. We have

$$
\mu_{j, A}=\int_{-\infty}^{\infty} R\left(\frac{\xi}{A}\right)\left|\hat{\psi}_{j, A}(\xi)\right|^{2} d \xi
$$

Pick a $\Delta>0$. It follows from (4) and (5) that for $A$ sufficiently large we shall have

$$
\hat{R}\left(\frac{\xi}{A}\right) \leqq 1-\frac{\Delta^{\alpha}}{2 A^{\alpha}}, \quad|\xi|>\Delta,
$$

and, by Lemma 6 , 


$$
\mu_{j, A} \geqq 1-\frac{2}{\lambda_{j} A^{\alpha}}
$$

Therefore

$$
\begin{aligned}
1-\frac{2}{\lambda_{j} A^{\alpha}} & \leqq \int_{-\Delta}^{\Delta}\left|\hat{\psi}_{j, A}(\xi)\right|^{2} d \xi+\left(1-\frac{\Delta^{\alpha}}{2 A^{\alpha}}\right) \int_{|\xi|>\Delta}\left|\hat{\psi}_{j, A}(\xi)\right|^{2} d \xi \\
& =1-\frac{\Delta^{\alpha}}{2 A^{\alpha}}+\frac{\Delta^{\alpha}}{2 A^{\alpha}} \int_{-\Delta}^{\Delta}\left|\hat{\psi}_{j, A}(\xi)\right|^{2} d \xi, \\
\int_{-\Delta}^{\Delta}\left|\hat{\psi}_{j, A}(\xi)\right|^{2} d \xi & \geqq 1-\frac{4}{\lambda_{j} \Delta^{\alpha}} .
\end{aligned}
$$

Since $\psi_{j, A}$ converges weakly to $f, \hat{\psi}_{j, A}$ converges boundedly to $\hat{f}$, so

$$
\int_{-\Delta}^{\Delta}|\hat{f}(\xi)|^{2} d \xi \geqq 1-\frac{4}{\lambda_{j} \Delta^{\alpha}}
$$

Since this holds for all $\Delta$ we have

$$
\int_{-\infty}^{\infty}|\hat{f}(\xi)|^{2} d \xi \geqq 1
$$

and the mean square convergence is established.

Let $g \in L_{2}$ be such that $|\xi|^{\alpha / 2} \hat{g} \in L_{2}$. We have

$$
A^{\alpha}\left(1-\mu_{j, A}\right)\left(\psi_{j, A}, g\right)=\int_{-\infty}^{\infty} A^{\alpha}\left(1-R\left(\frac{\xi}{A}\right)\right) \hat{\psi}_{j, A}(\xi) \hat{g}(\xi)^{*} d \xi .
$$

Choose a subsequence of $A$ 's so that

$$
\lim _{A \rightarrow \infty} A^{\alpha}\left(1-\mu_{j, A}\right)=\Lambda \text {. }
$$

(Observe that by Lemma $6 \Lambda \leqq \lambda_{j}^{-1}$.) Then the left side of (21) approaches $\Lambda(f, g)$. As for the right side, note that the functions

$$
\left\{A^{\alpha}\left(1-R\left(\frac{\xi}{A}\right)\right)\right\}^{1 / 2} \hat{\psi}_{j, A}(\xi)
$$

have uniformly bounded $L_{2}$ norm since

$$
\int_{-\infty}^{\infty} A^{\alpha}\left(1-R\left(\frac{\xi}{A}\right)\right)\left|\hat{\psi}_{j, A}(\xi)\right|^{2} d \xi=A^{\alpha}\left(1-\mu_{j, A}\right) \leqq 2 \Lambda
$$

for sufficiently large $A$. Thus we can find a subsequence of $A$ 's so that (22) converges weakly. Since it converges everywhere to $|\xi|^{\alpha / 2} \hat{f}(\xi)$ it follows, 
using Egoroff's theorem, that it converges weakly to $|\xi|^{\alpha / 2} \hat{f}(\xi)$. But since $|\xi|^{\alpha / 2} \hat{g}(\xi) \in L_{2}$,

$$
\lim _{A \rightarrow \infty}\left\{A^{\alpha}\left(1-R\left(\frac{\xi}{A}\right)\right)\right\}^{1 / 2} \hat{g}(\xi)=|\xi|^{\alpha / 2} \hat{g}(\xi) \quad \text { (mean square). }
$$

Thus (21) gives

$$
\Lambda(f, g)=\int_{-\infty}^{\infty}|\xi| \alpha \hat{f}(\xi) \hat{g}(\xi)^{*} d \xi
$$

It follows from (23) and Fatou's lemma that

$$
\int_{-\infty}^{\infty}|\xi|^{\alpha}|\hat{f}(\xi)|^{2} d \xi<\infty
$$

Put $g=\phi_{l}(l=1,2, \cdots)$ in (24). From (25) and Lemma 5 we obtain

$$
\Lambda\left(f, \phi_{l}\right)=\lambda_{l}^{-1}\left(f, \phi_{l}\right) \text {. }
$$

It follows from the completeness of the $\phi$ 's that $f=\phi_{l}$ (apart from a multiplicative constant) and $\Lambda=\lambda_{l}^{-1}$ for some $l$. It remains to show that we may take $l=j$, i.e., that $\lambda_{l}=\lambda_{j}$. By Lemma 6 we have $\lambda_{l} \geqq \lambda_{j}$. On the other hand since $\psi_{j, A}$ is orthogonal to $\psi_{k, A}(k=0, \cdots, j-1), f$ is orthogonal to $\phi_{k}$. Thus $\lambda_{l} \leqq \lambda_{j}$.

Lemmas 6 and 7 give the theorem.

\section{REFERENCES}

1. M. Kac, W. L. Murdock, and G. Szegö, On the eigenvalues of certain Hermitian forms, J. Rational Mech. Anal. vol. 2 (1950) pp. 375-384.

2. E. C. Titchmarsh, Introduction to the theory of Fourier integrals, Oxford, Clarendon Press, 1937.

3. S. V. Parter, On the extreme eigenvalues of truncated Toeplits matrices, Bull. Amer. Math. Soc. vol. 67 (1961) pp. 191-196.

4. H. Widom, On the eigenvalues of certain Hermitian operators, Trans. Amer. Math. Soc. vol. 88 (1958) pp. 491-452.

5. - Stable processes and integral equations, Trans. Amer. Math. Soc. vol. 98 (1961) pp. $430-449$.

CORNell University, ITHACA, NEW YORK 